\title{
The Numerical Simulation of the Interior Flow of a Dual Orifice Centrifugal Nozzle
}

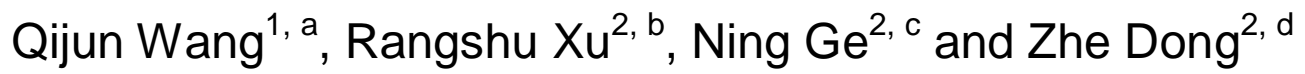 \\ ${ }^{1}$ Shenyang Institute of Special Equlpment Inspection \& research, Shenyang, China \\ ${ }^{2}$ Shenyang Aerospace University, Shenyang, Shenyang, China \\ a710085266@qq.com, bxurangshu@yahoo.com.cn, 610054817@qq.com, \\ ddongzhe233@126.com
}

Keywords: aero-engine; interior flow; CFD; VOF model; nozzle

Abstract. This paper attempts to research on a dual orifice centrifugal nozzle of a certain aero-engine using CFD method to numerically simulate the interior flow, which will provide reference for design.

The computing models for the primary fuel line and the secondary fuel line of the nozzle are built separately. The a reasonable computing domain is determined, and the geometry model is built using 3-D CAD software UG, and the grid of high quality is meshed by means of splitting volumes. With the boundary conditions and computing parameters determined according to data from experiments and references, the interior flow in the primary fuel line and the secondary fuel line of the nozzle is simulated using VOF model, and the velocity, pressure, phase volume fraction and spray cone angle are obtained for the primary and the secondary fuel line working separately. A 2-D axis-symmetrical model for the two circuits working simultaneously is created, with the result from the calculation of 3-D model as the boundary conditions, to give the spray pattern when working together for the primary and the secondary fuel line at the outlet of the nozzle. The comparisons between the numerical simulation results and the experiment data showed that the computing models comply with the nozzle working conditions.

\section{Introduction}

High-performance engine is inseparable from the performance of the combustion chamber, the nozzle as an important element on the combustion chamber, and its main role is broken into tiny droplets of fuel, according to different engine operating conditions, to a total number of suitable combustion chamber and it has a good fuel atomization quality. People through continuous exploration and research to get some experience formulas nozzle atomization. Appears CFD and matching computer software so that people can simulate the flow field inside the nozzle, which can be modified according to the internal nozzle flow. This topic is the use of computational fluid dynamics of the internal flow double centrifugal oil pressure atomizing nozzle is simulated.

At present, the main research and application of the nozzle is air atomizing nozzle and pressure atomizing nozzle. Pressure atomizing nozzle is the first aero engines commonly used, but pressure atomizing nozzle in high pressure ratio, high temperature or engine use when exposed some problems, such as in the high air pressure under the spray cone angle is smaller, the impact of the main combustion of the fuel distribution, increasing the smoke and flame radiation. Air atomizing nozzle appeared in the late 70's of twentieth Century, which is a major breakthrough in oil supply technology. The air atomizing nozzle ensures that the fuel oil and the gas flow are fully and evenly mixed, and the air atomizing nozzle has the advantages of low fuel supply pressure and the outlet temperature is not sensitive to the change of the fuel oil. So it has been widely used in high performance engine. 
However, the current aero-engine combustion chambers still have more high-performance engine with pressure atomizing nozzle, which is good because the pressure atomizing nozzle atomization quality, sophisticated design and experience, and has been continuously improved and perfected. Such as a double line pressure atomization at present widely good ignition performance and spray quality advantages in its stable combustion range is widely used in various types of engines. In addition, the modular air pressure atomizing nozzle atomization nozzle still in the applications.

The usual pressure atomizing nozzle is centrifugal. Centrifugal pressure nozzle according to the work principle and application, mainly divided into several: (1) a single pressure atomizing nozzle (Simplex Atomizer); (2) parallel type pressure atomizing nozzle atomizer (Duplex Atomizer ); (3) dual pressure atomizing nozzle Dual-orifice atomizer (Dual-orifice Atomizer); 4) overflow pressure atomizing nozzle atomizer (Spill Atomizer).

In China, the research of the pressure atomization nozzle is limited to the part of the production line application parameters, such as the circumferential non-uniformity and the spray cone angle of the fuel injection. For the flow field in the atomization quality is one of the most important size SMD (Sauter mean diameter), nozzle spout region, affecting the atomization quality factors are rarely studied. The simulation of the nozzle and the combustion chamber flame tube head, the research on the fuel injection trajectory and so on.

Since the 20th century 40's Arbor ramovic most early proposed discussion about centrifugal nozzle, scholars all over the world the many methods to study the centrifugal nozzle atomization process and spray parameters. Early research mainly based on experiment and theory analysis. With the development of computer technology, the use of computer simulation of the process of numerical simulation has gradually become more and more people pay attention.

Domestic and foreign scholars have used various models of centrifugal nozzle was simulated. Lixin Zhou etc. (2002) by a centrifugal nozzle parameters to simulate the flow field to obtain the nozzle parameters ${ }^{[1]}$; Ming Yue etc. (2003) with VOF method to capture the gas-liquid interface of the centrifugal nozzle ${ }^{[2]}$; Zhenguo Wang etc. (1996) adopted CFD method of gas-liquid centrifugal coaxial nozzle cold liquid mist to simulate two-phase flow smooth ${ }^{[3]}$. There are also many ways to scholars through experiments and theoretical analysis to calculate the nozzle flow were studied, Jun Chen etc. (2006) study by the method of trial nozzle atomization parameters ${ }^{[4]}$.

The practice shows that the simulation study of a certain type of engine nozzle in reducing load and cycle of work at the same time, but also help to the improvement of engine performance, deepen the combustor flow field, the study of the temperature field, thus greatly saving the test $\operatorname{cost}^{[5]}$.

This paper studies the fuel nozzle of a certain aero-engine combustion chamber. The nozzle is a double way double chamber centrifugal pressure atomizing nozzle. Spray model of upstream pressure and spray angle of two main parameters determined by the simulation and analysis of flow inside the nozzle of the main and auxiliary circuit. Then the two dimensional axisymmetric model is used to simulate the working conditions of the main and auxiliary oil circuit simultaneously.

\section{Basic equations of flow and physical model}

Inside the nozzle is a very complex turbulent motion. Neglecting heat exchange, turbulent flow in the nozzle is essentially three-dimensional, unsteady, multiphase, multicomponent, turbulent processes and their interactions. But these complex processes obey the basic governing equations of fluid motion, i.e. mass conservation equation, momentum conservation equation, and so on. At the same time, a variety of physical models need to be established in the simulation of the nozzle.

Basic flow equation. The general form of the mass conservation equation, also known as continuity 
equation, can be written:

$$
\frac{\partial \rho}{\partial t}+\frac{\partial}{\partial x_{j}}\left(\rho u_{j}\right)=S_{m}
$$

Wherein, $\rho$ is the density of the fluid; $u_{j}$ is fluid velocity direction of the $j ; j$ denote $\mathrm{x}, \mathrm{y}, \mathrm{z}$ directions; $S_{m}$ is the unit of time into the fluid mass of micro unit.

According to the law of conservation of momentum, momentum conservation equations can be expressed as

$$
\frac{\partial}{\partial t}\left(\rho u_{i}\right)+\frac{\partial}{\partial x_{j}}\left(\rho u_{i} u_{j}\right)=-\frac{\partial p}{\partial x_{i}}+\frac{\partial \tau_{i j}}{\partial x_{j}}+g_{i}-f_{i} .
$$

In the formula, $p$ is the pressure; $g_{i}$ and $f_{i}$ are gravity and other resistance components in $i$ direction; $\tau_{i j}$ is viscous stress tensor.

The flow inside the nozzle Reynolds number exceeds the critical value, the $\mathrm{k}$ - e equation turbulence model is used in the calculations. The $\mathrm{k}$ - model includes the standard k- e model, RNG k- model and Realizable k- model three. The internal flow of the nozzle has a lot of recirculation zone, and the size of the recirculation zone is very different. Taking into account the accuracy of our calculations and the existing computer resources, we choose to use the Realizable model.

Realizable $k-\varepsilon$ model is a new model developed recently. Standard $\mathrm{k}-\varepsilon$ model at strain rates are particularly large cases, it may lead to negative normal stress. In order to comply with the laws of physics of turbulent flow, the need for some kind of positive stress mathematical constraints. In order to ensure the realization of this constraint, the calculation formula of turbulent viscosity coefficient $\mathrm{C} \mu$ should not be constant, but should be linked to the strain rate, which appeared Realizable k- $\varepsilon$ model. It is better than the previous two models meet the Reynolds stress mathematical constraints and physical phenomena of turbulent flow more consistent.

In the Realizable $\mathrm{k}-\varepsilon$ model, $\mathrm{k}$ and $\varepsilon$ equations are:

$$
\begin{aligned}
& \frac{\partial}{\partial t}(\rho k)+\frac{\partial}{\partial x_{j}}\left(\rho k u_{j}\right)=\frac{\partial}{\partial x_{j}}\left[\left(\mu+\frac{\mu_{\tau}}{\sigma_{k}}\right) \frac{\partial k}{\partial x_{j}}\right]+G_{k}+G_{b}-\rho \varepsilon-Y_{M}+S_{k} . \\
& \frac{\partial}{\partial t}(\rho \varepsilon)+\frac{\partial}{\partial x_{j}}\left(\rho \varepsilon u_{j}\right)=\frac{\partial}{\partial x_{j}}\left[\left(\mu+\frac{\mu_{\tau}}{\sigma_{\varepsilon}}\right) \frac{\partial \varepsilon}{\partial x_{j}}\right]+\rho C_{1} E \varepsilon-\rho C_{2} \frac{\varepsilon^{2}}{k+\sqrt{v \varepsilon}}+G_{1 \varepsilon} \frac{\varepsilon}{k} C_{3 \varepsilon} G_{b}+S_{\varepsilon} .
\end{aligned}
$$

Among them, Wherein, $\mu_{t}$ turbulent viscosity; $G_{k}$ because the average velocity gradient caused by the generation of turbulence kinetic energy term; Gb buoyancy term is due to the generation of turbulence caused by the kinetic energy $k$; $\operatorname{Pr}$ is the turbulent Prandtl number of energy, taking 0.85; $\beta$ is the coefficient of thermal expansion; $Y_{M}$ contribution on behalf of compressible turbulent pulsation expansion; $M t$ turbulent Mach number; source term $S_{k}$ and $S_{\varepsilon}$ as user-defined. $C_{1 \varepsilon}, C_{2 \varepsilon}$ and $\mathrm{C} \mu$ are empirical constants; $\sigma_{k}$ and $\sigma_{\varepsilon}$ are the turbulent Prandtl number of $k$ and $\varepsilon$. These constant values in the literature have been published in recent years to reach consensus were $C_{1 \varepsilon}=1.44, C_{2 \varepsilon}$ $=1.92, \mathrm{C} \mu=0.09, \sigma_{k}=1.0, \sigma_{\varepsilon}=1.3$.

Turbulent flow in the near wall region is greatly affected by the wall. $K-\varepsilon$ model is mainly for high Re number of turbulent, it does not apply to the viscous sublayer, so in the near wall region must adopt a special approach to address the calculation of the flow near the wall.

The turbulent flow in the near wall region is greatly influenced by the wall surface. $K-\varepsilon$ model is mainly used in high Reynolds number turbulent calculation, does not apply to the viscous sublayer, therefore in the near wall region must adopt special methods, to solve the near wall flow 
calculation.

In most high Re number flow, the solution variable region near the wall changes very fast, often not necessary to solve it, so in the industrial flow simulation, the wall function of its economic advantages of more accurate calculations have been widely used. In this paper deal with the enhanced wall function.

Multiphase Flow Model. Multiphase turbulence model has VOF model, discrete phase model, hybrid model. Since the interior of the nozzle is substantially fuel flow flowing in the secondary air passage there will be the presence of vortices, the nozzle exit has a large amount of air, the air volume fraction is significantly greater than $20 \%$, it is suitable to use the Euler method is described. This paper used Euler method VOF method to calculate.

VOF model by solving the momentum equation and the volume fraction of each track on the entire fluid computational domain to simulate the flow of two or more immiscible fluids, the method is applicable to any two incompressible, do not mix, and between each other slip computational fluid negligible. The basic idea of VOF model is added to the model for each additional phase, it introduced a variable, that is, the phase in the volume fraction of the computing unit. Within each volume control all the volume fraction of the sum of 1 , all variables and properties shared between each phase and exhibit a volume average. As long as each phase at each position of the volume fraction known, the variable and physical properties in any particular unit or completely represents a phase, or on behalf of a mixture of phases, depending on the volume fraction values.

In the VOF model is accomplished by solving the continuity equation for the volume fraction of the interphase boundary tracking. For the phase $q$, the volume fraction equation has the following form

$$
\frac{1}{\rho_{q}}\left[\frac{\partial}{\partial t}\left(\alpha_{q} \rho_{q}\right)+\nabla\left(\alpha_{q} \rho_{q} \stackrel{r}{v}_{q}\right)\right]=S_{\alpha_{q}}+\sum_{p=1}^{n}\left(n \alpha_{p q}-n \alpha_{q p}\right) .
$$

Among them, $n \delta_{p q}$ is the quality that is passed from the $p$ phase to $q$ phase, $n \delta_{q p}$ is the quality that is passed from the $q$ phase to $p$ phase, while the source term on the right side of equation is $S_{\alpha_{q}}=0$ by default.

In the whole calculation domain, the single momentum equation is solved, and the velocity field is shared in each phase. The form of the momentum equation is shown below, which depends on the volume fraction of all phases.

$$
\frac{\partial}{\partial t}(\rho \stackrel{r}{v})+\nabla(\underset{\rho v v}{r r})=-\nabla p+\nabla\left[\mu\left(\nabla \stackrel{r}{v}+\nabla v^{r}\right)\right]+\rho \stackrel{r}{g}+\stackrel{r}{F}
$$

\section{Calculation model and model parameters}

As shown in Fig. 1, the centrifugal nozzle is composed of the main oil path and the auxiliary oil path. The main oil line has six weeks to the distribution of the cut to the tank and secondary circuit with three weeks to the distribution of the cutting to the tank, main and auxiliary fuel circuit respectively in 60 degrees cycle, to $120 \mathrm{DEG}$ to cycle to uniform distribution and therefore were taken $1 / 6$ of the main oil, $1 / 3$ of the secondary circuit for the computational domain. The exit boundary of the computational domain exits at a certain distance from the nozzle. The pressure is equal to infinity, and is defined as the pressure outlet boundary. Most of the area is divided into hexahedron mesh; tangential groove with hexahedral mesh. Using the method of setting interface to realize the non-uniform transition of the tangential slot and its two ends. The main oil path number 
is 390 thousand, and the auxiliary circuit is the main path. The wall $y+$ of the main and auxiliary oil path model is in the 1 5 range, which meets the requirement of wall surface $\mathrm{y}^{+}$.

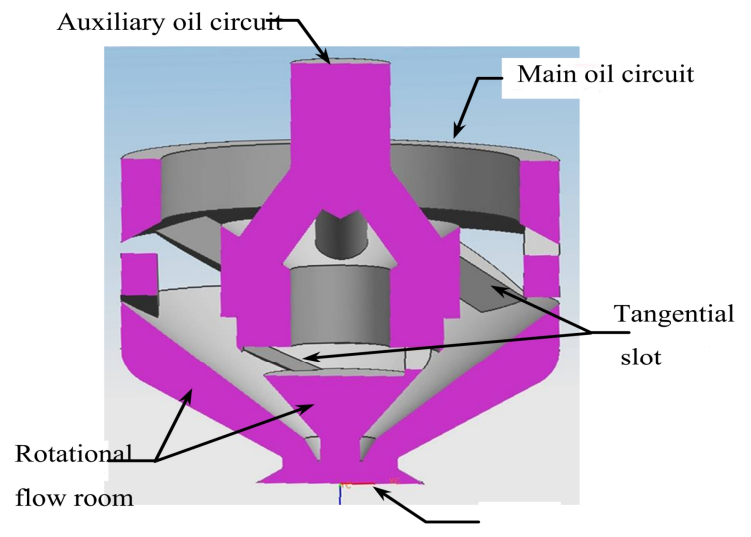

Fig. 1 Double circuits centrifugal atomizer

Fuel inlet boundary is set to speed inlet. Single nozzle of the main oil inlet mass flow $\mathrm{m}=$ $0.010743 \mathrm{~kg} / \mathrm{s}$, fuel density $\rho=800 \mathrm{~kg}$, by using $\rho v A=m$, the winner oil inlet velocity will be $\mathrm{v}=$ $2.565 \mathrm{~m} / \mathrm{s}$; similarly, can be obtained auxiliary fuel inlet velocity is $0.283 \mathrm{~m} / \mathrm{s}$. At the same time, the volume fraction of the second phase in the second phase of the main and auxiliary oil circuit is set to 1 .

\section{Calculation results and analysis}

Fig.2 and Fig.3 are, respectively, based on the volume fraction of fuel oil and fuel oil. In the swirl chamber of the main oil path, the fuel is basically filled with swirl chamber. Because most of the middle of the main oil flow in the middle of the secondary circuit is occupied by, so the main oil is basically full of fuel oil. In the secondary circuit can be seen as a result of the rotation of the fuel to the middle of the air vortex. The air vortex occupies a large part of the swirl chamber of the secondary oil path.

From Fig.2 and Fig.3 can be seen during the injection nozzle droplets crushing process, the fuel compressed into a liquid film in the cyclone chamber. After discharge spout due to the interaction of the air drawn into filaments, and then broken up into droplets.

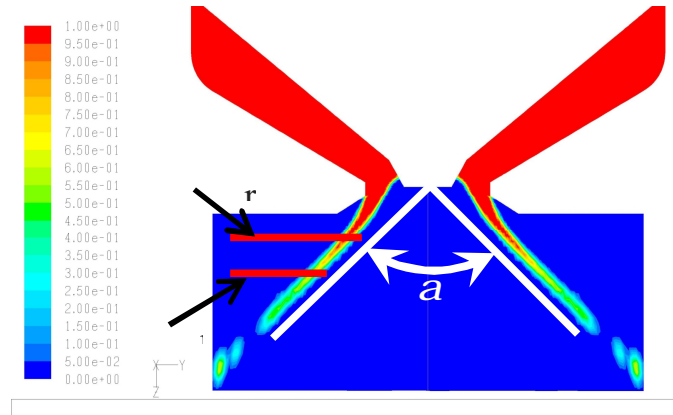

Fig. 2 Fuel volume fraction distribution of main oil path
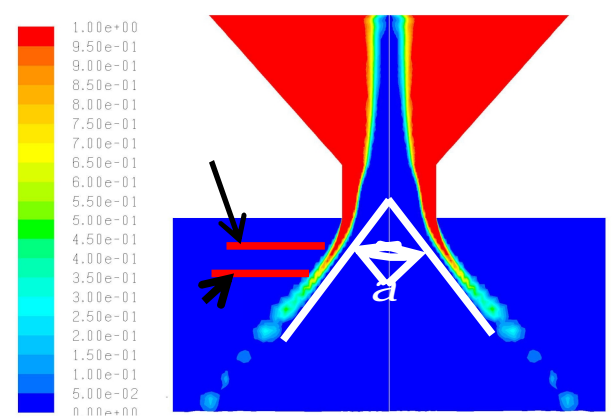

Fig.3 Volume fraction distribution of secondary oil path

Circumferential position of the computational domain in two radial nozzle exit radius circumference of $y$, along the direction of the injector nozzle exit in a straight line. Find them on the straight line fuel volume fraction of the maximum average $\mathrm{z}$ coordinates and radial radius $\mathrm{y}$, can be determined spray angle. 


$$
\alpha=2 \times \arctan \left|\frac{y_{1}-y_{2}}{z_{1}-z_{2}}\right| .
$$

Calculate the main oil spray angle of $\alpha=86.8^{\circ}$ Calculate the main oil spray angle of $\alpha=86.8^{\circ}$, deputy oil spray cone angle of $\alpha=78^{\circ}$, coincides with Chen Jun and other test results.

From the main oil flow trajectories to speed internal coloring (Fig.4) can be seen due to the contraction of the swirl chamber compressed air flow path and rotating the role of the nozzle orifice flow rate reaches the maximum tangential fuel tank to provide a tangential velocity, the tangential velocity can guarantee the fuel discharged at the nozzle exit cone away. Tangential velocity to the role of the fuel swirl outdoor wall centralized, thus making the wall near the cyclone outdoor place higher speed in the cyclone chamber. From the speed of flow traces can clearly see the rotation of the fuel in the swirl chamber flow down.

Calculation of the upper reaches of the main oil pressure is $2.84 \mathrm{MPa}$, the auxiliary circuit is $0.535 \mathrm{MPa}$. The calculated value of the pressure is in good agreement with the experimental results.

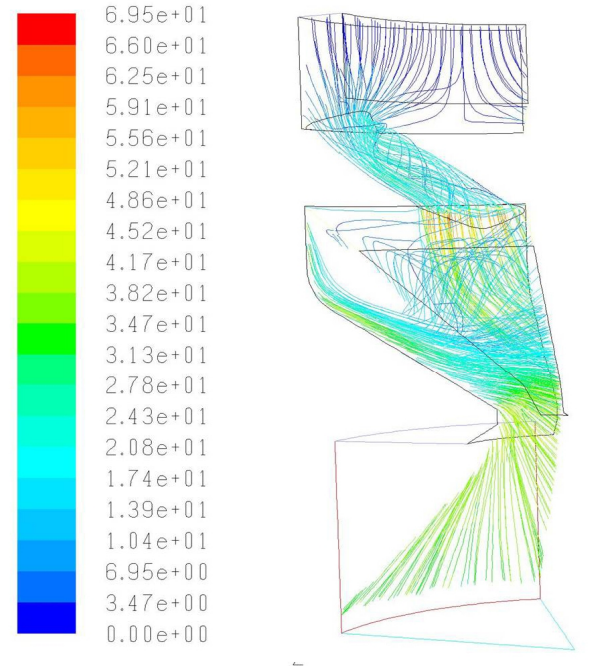

Pathlines Colored by Velocity Magnitude (mixt)

Fig.4 The main oil line to internal flow trajectories coloring speed

\section{Conclusion}

1. Using computational fluid dynamics method, on the two-phase flow inside the centrifugal nozzle to carry on the simulation computation, important characteristic parameters are consistent with the experimental results of the spray cone angle, fuel flow rate and pressure loss are obtained. For the nozzle design have great reference value.

2. VOF two-phase flow model can be used to solve the fuel and air flow in the nozzle.

3. The value of the flow inside the nozzle calculated using finer mesh wall $y+$ value is small, near-wall treatment methods should be used to enhance wall treatment

4. Initial iterative calculation sub regional initialization method can improve the stability of the iterative process, the speed of convergence.

\section{References}

[1] Lixin Zhou etc. A centrifugal nozzle parameters to simulate the flow field[J], Journal of Propulsion Technology,2002,23(6): 480-484

[2] Ming Yue etc. Numerical simulation of gas liquid two phase flow in a centrifugal nozzle[J], Journal of Engineering Thermophysics,2003,24(5): 888-890

[3] Zhenguo Wang etc. Numerical simulation of gas-liquid coaxial centrifugal nozzle spray flow field[J], Journal of Propulsion Technology,1996,17(3): 43-49

[4] Jun Chen etc. Experimental research on a certain type of Aeroengine Fuel nozzle[J], Gas Turbine Experiment and Research,2006,19(3): 40-43

[5] Zheng Zhang etc. Study on atomization characteristics of two way centrifugal nozzle[J], Journal of Engineering Thermophysics,2003,24(1):153-156 